\title{
Culture-dependent and Universal Constructs and Promoting Factors for the Process of Personal Recovery in users of Mental Health Services: Qualitative Findings from Japan
}

Akiko Kanehara ( $\nabla$ a-kanehara@umin.ac.jp )

University of Tokyo Hospital

Haruna Koike

University of Tokyo Hospital

Yumiko Fujieda

University of Tokyo Hospital

\section{Sayaka Yajima}

University of Tokyo Hospital

Asami Kabumoto

University of Tokyo Hospital

\section{Yousuke Kumakura}

University of Tokyo

Kentaro Morita

University of Tokyo Hospital

Yuki Miyamoto

University of Tokyo

Masahiro Nochi

University of Tokyo

Kiyoto Kasai

University of Tokyo

\section{Research Article}

Keywords: personal recovery, culture, qualitative research, Japan, mental illness

Posted Date: March 3rd, 2021

DOl: https://doi.org/10.21203/rs.3.rs-258956/v1

License: (9) This work is licensed under a Creative Commons Attribution 4.0 International License.

Read Full License 
Version of Record: A version of this preprint was published at BMC Psychiatry on February 10th, 2022. See the published version at https://doi.org/10.1186/s12888-022-03750-4. 


\section{Abstract}

Background: The conceptualization of personal recovery began in Europe and North America and has spread worldwide. The concept of personal recovery and recovery-promoting factors may be influenced by culture. We explored how users of mental health services in Japan perceive their own personal recovery and the factors that promote it.

Methods: We conducted semi-structured interviews and focus group interviews with individuals using mental health services. The interview data underwent thematic analysis, and we adopted a grouped framework analysis approach. We used a coding framework based on the existing CHIME framework (connectedness, hope and optimism about the future, identity, meaning in life, and empowerment). We counted the number of narratives for each element of the framework.

Results: Data were obtained from 30 users of mental health services (mean age: 40.4 years; $46.7 \%$ women; $50.0 \%$ with schizophrenia). "Compassion for others" was newly extracted in "Connectedness", and "Rebuilding/redefining identity not being as shaped by social norms" was newly extracted in "Identity" as personal recovery. "Positive experiences in childhood" (including positive parenting support from neighbours) was newly extracted as a recovery-promoting factor. Connectedness was most frequently coded in our study.

Conclusions: Our unique findings of the rebuilding/defining identity free from conformity to social norms through communications with relatively familiar people including peers may be culture dependent. This study raises overarching questions regarding how socio-cultural values influence the development of identity and personal values and how they are in turn reflected in personal recovery.

\section{Introduction}

Personal recovery is a unique process of changing one's attitudes, values, feelings, goals, skills, and/or role and developing new meaning in life beyond illness [1]. Based on their systematic review of the literature, Leamy et al. [2] described a conceptual framework of personal recovery that includes the following elements: connectedness; hope and optimism about the future; identity; meaning in life; and empowerment (CHIME) [2]. The CHIME recovery framework led to the development of a questionnaire about the process of recovery (QPR) [3, 4], which was later translated into Chinese [5], Swedish [6], and Japanese $[7,8]$.

Regarding the recovery-promoting factors, multiple items have been identified in qualitative studies and literature reviews. For example, one systematic review indicated that recovery-promoting factors included (1) adjustment, coping, and reappraisal; (2) responding to the illness; and (3) social support, close relationships, and belonging [9]. Another systematic review identified five factors: (1) social support, (2) faith and spirituality, (3) personal agency and hope, (4) environmental resources, and (5) positive support and holistic care from services [10]. 
An individual's culture adds another dimension to the concept of personal recovery and recoverypromoting factors. For example, a systematic literature review of Nordic research on personal recovery indicated the existence of a need to identify the process of recovery that reflected the Nordic mental health care systems [11]. Several studies have discussed cultural differences in the conceptualization of personal recovery [12-14]. Specifically, one literature review showed that people from Black and minority ethnic backgrounds emphasized spirituality and stigma in their recovery, and it also identified themes such as cultural facilitating factors and collectivist notions of recovery [2]. The available literature is limited on the conceptualization of personal recovery and the factors that promote it in Asian countries, including Japan. A review of Asian perspectives on personal recovery in mental health showed that support from family, friends, and social connections was the most salient recovery-promoting factor, while religious stigma, discrimination, gendered norms, and negative societal perceptions of mental illness hindered recovery [15].

Understanding culture-specific concepts of personal recovery and recovery-promoting factors is essential for recovery-oriented support. However, little is known regarding these conceptualizations in Japan. In this study, we aimed to explore (1) the concept of personal recovery and (2) factors that promote recovery among users of mental health services in Japan.

\section{Methods}

All methods were carried out in accordance with the Consolidated criteria for reporting qualitative research (COREQ) checklist [16] (Additional file 1). We also conducted this study with reference to qualitative research guidebooks [17-21]

\section{Study designs}

The study involved the use of the semi-structured interviews and focus group interviews that focused on people's experience of the process of recovery.

\section{Participants}

We conducted interviews with users of mental health services in Japan. Inclusion criteria were as follows: (1) age $\geq 16$ years, (2) ability to participate and give informed consent, and (3) use of mental health services.

For study participants under the age of 20 , informed assent was obtained from the participants and written informed consent was obtained from their parents.

We included participants with any mental health difficulties because the concept of personal recovery involves a person who has experienced mental health difficulties and it tends to be transdiagnostic. In addition, previous studies on personal recovery did not limit the diagnosis [2, 22]. In our survey, the participants self-reported their diagnosis from a list of diagnostic categories, which included the categories 'other' and 'not known'. We continued data collection until reaching theoretical saturation, 
which is defined as the point at which researchers have gathered enough data such that more sampling will not provide more information related to their research questions [23].

We recruited the participants from five community-based mental health services in urban, suburban, and rural communities in Japan.

In the first step of the recruitment process, mental health staff talked to service users about the study and showed them flyers that explained its aims and methods.

The flyers also indicated that participants would be given 5,000 Japanese Yen (JPY) (equivalent to about 40 EUR or US\$45) for participation.

Next, we explained the details of the study to interested individuals, and those who agreed to participate provided written informed consent.

Participation was voluntary, and participants had the option to discontinue if they felt distressed or otherwise did not wish to continue with the study.

\section{Data collection}

After reviewing the relevant literature on qualitative interviews, testing a pilot interview guide and discussing them with experts in qualitative research, we created a semi-structured interview guide. By referring to a previous study [24], we decided to ask the participants about their process of recovery. The first interview question was, "Have there been any recent changes since you experienced mental illness?" Our intent was to focus on recent positive changes, not negative changes that occurred at the time of onset of the mental health illness.

Regarding the recovery-promoting factors, we asked participants to identify factors that influenced the experience and process.

The interview included the following questions, with questions 4 and 5 focusing on recovery-promoting factors in particular:

1. Was there any recent change since you experienced mental illness?

2. Is there anything you have gained from experience?

3. Please describe your recovery in a word?

4. Is there any experience or value that influenced your process of recovery?

5. Is there anyone who has influenced your process of recovery?

The interviews were conducted by the first author (AK). AK was a female PhD student who has worked as a clinical and research social worker in mental health settings. AK was not involved in the clinical care of any of the participants. AK has a special interest in the process of personal recovery.

None of the participants asked about the interviewer's occupational background.

All interviews were audio-recorded and transcribed. Data were collected between June 2017 and October 2017. 
Individual and focus group interviews were used because having multiple data sources in qualitative research (triangulation) improves the reliability of the results and enriches the quality of the information obtained [25].

We explained the characteristics of the individual and focus group interviews to the participants and asked them to select which type of interview they would like to do.

The interviews ( 60 minutes) and focus group interviews (90 minutes) were conducted at the convenience of the participants (e.g.

clinics, a hospital and a mental health community facility).

We also collected quantitative data from the participants through three self-report questionnaires. The Questionnaire about the Process of Recovery (QPR) was developed based on the CHIME recovery framework with 22 items [3] and translated into Japanese [7, 8]. The WHO 5 Well-being Index (WHO-5) measures current mental well-being with five questions [26, 27], and we used the Japanese version developed previously [28-30]. The WHO Disability Assessment Schedule 2.0 (WHODAS 2.0) is a questionnaire that was developed to assess health and disability across different cultures and settings. It encompasses six domains of functioning (cognition, mobility, self-care, getting along, life activities, and participation) [31], and it was previously translated into Japanese [32].

All participants received 5,000 Japanese Yen (JPY) (equivalent to about 40 EUR or US\$45) for each interview.

AK made field notes after the interviews.

\section{Analysis}

The interviews and our analysis were conducted in Japanese, and we subsequently translated the results into English. A thematic analysis was conducted, and a grouped framework analysis approach was adopted [33], using the following process. First, the first author (AK) and a licensed clinical psychologist (HK) independently read the transcripts several times to familiarize themselves with the data. Second, AK and HK individually coded each line of the transcripts. Finally, AK and HK clustered and synthesized codes to form themes.

Our coding framework was based on the existing CHIME framework [2]. This coding framework was previously used in a systematic review that described international differences in the concept of personal recovery [13]. We used the descriptions of recovery process categories from the existing CHIME framework, which was created based on research by Leamy et al. [2] (Additional file 2: Table S1) and a review by Slade et al. [13]. We also classified the recovery-promoting factors according to the subject of factors (the participant, others, and medical) and the CHIME framework. We also summarized the selected narratives from the interviews to describe subthemes.

Coding was done according to the coding framework, but our methodology allowed identification of new additional themes in the interview data. After the themes were identified, the frequency of each theme's appearance in the transcripts was calculated. We counted the number of narratives for each theme of the 
framework. If one narrative fit multiple themes, it was counted in each. Throughout the analysis, crosschecking and discussion with research team members were utilized to validate that the themes were meaningful and to ensure the data analysis was reliable.

All authors reviewed the data and checked themes and conclusions. The research team included clinicians (psychiatrists, psychologists, and an occupational therapist) and researchers (qualitative study, nursing, and public health).

The Ethical Committee of the Faculty of Medicine, The University of Tokyo approved this study [approval No. 11506].

\section{Results}

\section{Participant Characteristics}

We continued the data collection until we confirmed that we had sufficient data to account for all aspects of the themes. Data collection ended after we had interviewed and analysed data from 30 participants. Of these 30 people, 15 participated in an individual semi-structured interview, and 15 participated in the focus group interviews. The demographic characteristics of the 30 participants (mean age: 40.4 years; $46.7 \%$ women; $50.0 \%$ with schizophrenia) are shown in Table 1 . The average interview time was 48.3 minutes for the interviews and 65.5 minutes for the focus group interviews.

We present our results in terms of the concept of personal recovery and the recovery-promoting factors.

\section{Concept of personal recovery}

We used a coding framework based on the existing CHIME framework [2] (Additional file 2: Table S1). Coding was done according to the framework, and coders could identify additional themes in the interview data as needed. No new framework was extracted from the data, but new categories were extracted and fell within the existing framework. "Compassion for others" was newly extracted in "Connectedness", and "Rebuilding/redefining identity not being as shaped by social norms" was newly extracted in "Identity". The concept of personal recovery and selected narratives from the interviews are summarized in Table 2a.

\section{Compassion for others in Connectedness}

Through the experience of mental illness and difficulties, the participants described that they were able to imagine others' circumstances, including difficult situations, and to accept people with different values. A greater ability to have compassion with others was identified.

I became kinder and had more compassion for others than I did before I experienced illness. 
I became able to image the background of others and accept diversity.

I want to understand the feelings of people who are suffering from difficulties and want to make use of that experience in my work.

I used to believe that mental illness was just being lazy, but through my own experience, I understood that mental illness was not laziness but illness, and I found it painful. I could be generous to myself and others.

\section{Rebuilding/redefining identity not being as shaped by social norms in Identity}

Participants who experienced mental illness and difficulties, moved away from the social norms of their group (company/school) and their original identities and values changed. They redefined their identity as being less shaped by the expectations of social norms.

\section{Social norms that valueacademic success}

I felt that I suffered from illness because of an overemphasis on educational qualifications. Treatment liberated the thought.

\section{Social norms that value diligence or productivity}

I started to think that errors and unpredictable things are interesting.

I did not doubt that hard work, good grades, and getting a good job are necessary for wonderful life. But I had no friends. I had the experience of illness and made many friends.

I used to work hard and worked to the limit. Right now, I am consciously resting and not working too hard. (C2, 14, E1, E2)

I used to lose myself in a place where productivity was the top priority, but now I have a place to play my role. $(\mathrm{C} 2,14, \mathrm{M} 1, \mathrm{M} 4)$

I come to think that I want to stay as I am. $(12,14)$

\section{Counting of the concept of personal recovery}

After the themes were coded, counting was conducted to calculate how frequently each theme appeared in the interviews. We counted the number of narratives for each of the categories within the framework 
(Table 2b). Connectedness was most frequently coded in our study (35.2\%).

\section{Recovery-promoting factors}

The recovery-promoting factors (the participant, others, and medical) and CHIME framework are summarized in Additional file 3: Table S2.

\section{Positive childhood experiences}

The existence of positive experiences in childhood (including positive parenting support from neighbours) was newly extracted as a recovery-promoting factor.

My parents raised me to believe in me. I have accepted it and have lived. That encourages recovery.

My neighbour has helped me since my childhood. After I had a mental illness, the neighbour has helped me with housing and working.

\section{Respectful communication from professionals}

In psychiatric care, not only were specific therapies important, but respectful communication by professionals also promoted personal recovery. For example, "unconditional positive regard" by the professionals and "support to discuss not only symptoms but also everyday life and enjoyment" were emphasized.

My parents only disapproved of me, but the counsellor accepted me unconditionally.

I was able to talk to my supporter about my daily life. She did not limit my enjoyment and supported me.

\section{Communication from peers}

Communication with peers who also experienced mental illness was another recovery-promoting factor. Such communication included interactions that promoted rebuilding an identity that is not shaped by social norms, or helped deconstruct social norms.

When I felt sad that I had an illness I never wanted to have, a person who had also experienced a mental illness gave me a warm smile and warm comments.

I was career-oriented, but a peer taught me to enjoy everyday life.

I had thought that my efforts would improve the situation, but my efforts did not improve my mental illness. I was told that I was good as I was and realized that it is better to rely on medicines and peers. 


\section{Support from families}

Family attitudes such as unconditional positive regard and caring communication were extracted as recovery-promoting factors.

My family remained calm even though I was confused and emotional and had been with me for a long time, even when my condition was severe. It gave me a sense of security that my family wouldn't abandon me.

\section{Respectful communication from acquaintances}

Words and attitudes from colleagues or friends were extracted.

A colleague also understood my illness and treated me kindly.

Talking to my colleagues about my condition, I was able to work in a way that fits my condition.

My friend did not focus on my illness, and I thought that he was happy to be with me, and he talked to me and treated me as usual.

\section{Self-learning/Physical activity}

Self-learning and physical activity were also extracted as recovery-promoting factors.

I was exposed to different values in movies and TV dramas. (People with mental illness are usually working, people living in community meet people with illnesses every day, people consider physical and mental disorders to be the same)

I thought that mental illness was scary and unfamiliar. I understand that mental illness can be scientifically explained.

I was running to build physical strength, deal with loneliness, and improve motivation. Running facilitated recovery.

\section{Discussion}

To identify culture-dependent and universal constructs and factors promoting the process of personal recovery, we conducted individual and focus group interviews with users of mental health services in Japan. We then undertook a thematic analysis of the interview data. Our findings revealed that the CHIME personal recovery concept is generally relevant to people living in Japan. "Compassion for others" was newly extracted in "Connectedness", and "Rebuilding/redefining identity not being as shaped by social norms" was newly extracted in "Identity". Connectedness was the most frequently coded category in our 
study. The presence of positive experiences in childhood (including positive parenting support from neighbours) was newly extracted as a recovery-promoting factor.

\section{Concept of personal recovery}

\section{Compassion for others in Connectedness}

The theme of "compassion for others" was not described in the original CHIME framework and was newly extracted in our study. Our finding is compatible with a recent study by Slade and colleagues [34] of posttraumatic growth for people with psychosis and other severe mental health problems [34]. Through experiencing the frustration, suffering, and pain associated with their mental disorders, the service users were able to think of others' emotions, thoughts, and backgrounds; accept a wide range of values; and have compassion for others. The painful experience of having an often stigmatized mental illness diagnosed and the associated social disadvantages may have made them feel compassionate toward others, especially those with a minority status.

\section{Rebuilding/redefining identity not being as shaped by social norms in Identity}

In a 33-nation study that revealed differences between a "tight culture" (with strong norms and low tolerance of deviant behaviour) and a "loose culture" (with weak norms and a high tolerance of deviant behaviour), Japan was identified as a relatively tight culture [35]. In our study, a person who had social norms that valued "hard work, good grades, and getting a good job" encountered a person or peer with different values. By becoming acquainted with the new values, they eased up on their adherence to the social norms that they originally followed and came to value having friends and enjoying everyday life. During the recovery process, a person who originally had many relatively strong social norms would have encountered peers and peer support workers who were not bound by the same social norms, and the person subsequently formed identities that did not depend on their previous social norms.

\section{Connectedness was coded the most frequently in our study.}

Many Asian cultures place value on fitting in and on harmonious interdependence with others. In a study on the development and validation of attitudes towards recovery questionnaire among Chinese people, the authors emphasized "family involvement" as one of the attitudes influencing personal recovery in the Chinese context [36]. In American culture, individuals seek to maintain their independence from others by discovering and expressing their unique inner attributes [37]. Among Japanese and Filipino populations, perceived emotional support positively predicted subjective well-being even after self-esteem was controlled for [38]. However, among Euro-Americans, perceived emotional support weakly predicted 
subjective well-being, and moreover, the association disappeared once self-esteem was statistically controlled for [38]. Another study indicated that individualistic values were negatively related to interpersonal relationships and subjective well-being for Japanese college students, but not for American college students [39]. The results of the previous studies are consistent with "connectedness" being the most frequently coded factor in our study.

In Japan, connectedness with familiar people such as family members, friends, and colleagues was extracted from the data, and the "Being part of the community" was not coded. Due to the influence of their focus on community integration in the US and social inclusion in the UK, "Being part of the community" was frequently coded [13]. This difference between Japan and other countries may partly stem from insufficient community support in Japan [40].

\section{Recovery-promoting factors}

Previous studies indicated that recovery-promoting factors include close relationships, social support, and positive support from mental health services $[9,10]$. In addition to those themes, the theme of positive childhood experiences was extracted in our study.

\section{Positive childhood experiences}

A previous study showed that positive childhood experiences might reduce the risk for adult depression and poor mental health, as well as promote adult relational health [41]. In our study, the internalization of positive childhood experiences directly or indirectly influenced the promotion of personal recovery. Previous personal recovery studies may have focused on external resources and not accounted for the human capital stored within individuals. We need to focus on aspects of human capital (skills, abilities, experience, motivation, intelligence, health, and productivity) that contribute to personal recovery and well-being [42].

\section{Professionals' respectful communication}

Communication with professionals appeared as one of the recovery-promoting factors. A previous study showed that providers' respectful communication was associated with personal recovery from mental health problems [43]. Respectful communication may play a key role in facilitating personal recovery through avenues such as empowerment and hope/confidence.

\section{Communications with peers}

Peers with similar experiences contributed to personal recovery in ways such as reconstructing experiences, rebuilding relationships with others, and finding meaning in life. A previous review showed 
that peer support workers could foster hope and belief in the possibility of recovery, including empowerment, increased self-esteem, self-efficacy, self-management, social inclusion, engagement, and increased social networks [44]. Our results also indicated that communications with peers facilitated rebuilding an identity that is not as shaped by social norms or helping to deconstruct social norms.

\section{Strength and limitations of this study}

Our analysis involves a team of clinicians (psychiatrists/psychologists) and researchers (psychology/nursing/public health) to improve the reliability of data analysis. However, several limitations of this study warrant consideration. First, some of the study participants were recruited from community mental health service organizations known for excellent user-centred service. This might account for the high proportion of positive experiences during the process of recovery compared with the experiences of individuals using standard care services in Japan. Second, our study did not consider the duration or severity of the participants' disorders. Third, this study was led by researchers and clinicians. The co-production of research (full involvement in research by people with mental illness) is warranted for future studies [45].

\section{Implications}

The conceptualization of personal recovery and identification of the factors that promote it provide a theoretical foundation for changing attitudes to support recovery in the mental health field. In clinical settings, respectful communication of medical staff is important for promoting personal recovery. Communication with familiar people, including peers who help users of mental health services free themselves from conformity to social norms, might be important for personal recovery in the Japanese culture.

Our study found that the major facilitators for the process of personal recovery includes daily natural and intentional support for rebuilding identity by peers and peer support workers. These results have important implications for organizational change in the medically oriented and professional-led mental health service system.

\section{Conclusion}

In conclusion, our study demonstrated the constructs for personal recovery and the promoting factors in users of mental health services in Japan. Most of the constructs in the CHIME framework were replicated and thus may be regarded as universal. In contrast, our unique findings of rebuilding identity free from conformity to social norms through communications with familiar people, including peers, may be culture dependent. This study from a far-eastern country may posit a more universal question of how the development of identity and personal values $[46,47]$ are influenced by socio-cultural values and how they are in turn reflected in an individual's recovery journey. 


\section{Abbreviations}

QPR: The questionnaire about the process of recovery; WHO-5: The World Health Organisation Five WellBeing Index; WHO-DAS2.0: The World Health Organization Disability Assessment Schedule 2.0; COREQ: Consolidated criteria for reporting qualitative research.

\section{Declarations}

\section{Acknowledgements}

The authors express their gratitude to Drs. Mike Slade, Jane McGregor, and Geoff Shepherd for their kind introduction to the concept of personal recovery and recovery-oriented service.

\section{Authors' contributions}

A Kanehara, YK, YM, MN and KK made substantial contributions to conception and design. A Kanehara, HK, YF, SY, A Kabumoto and KM made substantial contributions to acquisition of data. All authors made substantial contributions to analysis and interpretation of data. A Kanehara, HK, YF, SY and A Kabumoto have been involved in drafting the manuscript. $\mathrm{YK}, \mathrm{KM}, \mathrm{YM}, \mathrm{MN}$ and $\mathrm{KK}$ have been involved in revising it critically for important intellectual content. All authors given final approval of the version.

\section{Funding}

This study was partially supported by JSPS KAKENHI Grant Number JP16H06395, JP16H06398, JP16H06399, JP16K21720, and 20H03596. This research was also partially supported by Research and Development Grants for Comprehensive Research for Persons with Disabilities from Japan Agency for Medical Research and Development, AMED (Grant Number: 16dk0307066 and 16dk0307059). This work was supported by MHLW DA Program Grant Number JPMH20DA1001. This work was also supported in part by UTokyo Center for Integrative Science of Human Behavior (CiSHuB), and by the International Research Center for Neurointelligence (WPI-IRCN) at The University of Tokyo Institutes for Advanced Study (UTIAS). The funders had no role in the design of the study; the collection, analysis, and interpretation of data; or the writing of the manuscript.

\section{Availability of data and materials}

The dataset supporting the conclusions of this article is not available due to ethical restrictions.

\section{Ethics approval and consent to participate}


The Ethical Committee of the Faculty of Medicine, The University of Tokyo approved this study [approval No. 11506]. All participants provided written informed consent.

\section{Consent for publication}

Not applicable.

\section{Competing interests}

The authors declare no conflict of interest.

\section{References}

1. Anthony WA. Recovery from mental illness: The guiding vision of the mental health service system in the 1990s. Psychosoc Rehabil J. 1993;16(4):11-23.

2. Leamy M, Bird V, Le Boutillier C, Williams J, Slade M. Conceptual framework for personal recovery in mental health: systematic review and narrative synthesis. Br J Psychiatry. 2011;199(6):445-52.

3. Neil S, Pitt L, Kilbride M, Welford M, Nothard S, Sellwood W, et al. The questionnaire about the process of recovery (QPR): a measurement tool developed in collaboration with service users. Psychosis. 2009;1(2):145-55.

4. Shanks V, Williams J, Leamy M, Bird VJ, Le Boutillier C, Slade M. Measures of personal recovery: a systematic review. Psychiatr Serv. 2013;64(10):974-80.

5. Chien WT, Chan ZC. Chinese translation and validation of the questionnaire on the process of recovery in schizophrenia and other psychotic disorders. Res Nurs Health. 2013;36(4):400-11.

6. Argentzell E, Hultqvist J, Neil S, Eklund M. Measuring personal recovery-psychometric properties of the Swedish Questionnaire about the Process of Recovery (QPR-Swe). Nord J Psychiatry. 2017;71(7):529-35.

7. Kanehara A, Kotake R, Miyamoto Y, Kumakura Y, Morita K, Ishiura T, et al. The Japanese version of the questionnaire about the process of recovery: development and validity and reliability testing. BMC Psychiatry. 2017;17(1):360.

8. Kanehara A, Kotake R, Miyamoto Y, Kumakura Y, Morita K, Ishiura T, et al. The Japanese version of the questionnaire about the process of recovery: development and validity and reliability testing. BMC Psychiatry. 2017;17(1):360. [Correction: BMC Psychiatry. 2020;20(1):12.]

9. Soundy A, Stubbs B, Roskell C, Williams SE, Fox A, Vancampfort D. Identifying the facilitators and processes which influence recovery in individuals with schizophrenia: a systematic review and thematic synthesis. J Ment Health. 2015;24(2):103-10.

10. Wood L, Alsawy S. Recovery in psychosis from a service user perspective: a systematic review and thematic synthesis of current qualitative evidence. Community Ment Health J. 2018;54(6):793-804. 
11. Schön UK, Rosenberg D. Transplanting recovery: research and practice in the Nordic countries. J Ment Health. 2013;22(6):563-9.

12. Tse S, Davidson L, Chung KF, Ng KL, Yu CH. Differences and similarities between functional and personal recovery in an Asian population: a cluster analytic approach. Psychiatry. 2014;77(1):41-56.

13. Slade M, Leamy M, Bacon F, Janosik M, Le Boutillier C, Williams J, et al. International differences in understanding recovery: systematic review. Epidemiol Psychiatr Sci. 2012;21(4):353-64.

14. Slade $M$, Amering $M$, Farkas $M$, Hamilton $B$, O'Hagan $M$, Panther $G$, et al. Uses and abuses of recovery: implementing recovery-oriented practices in mental health systems. World Psychiatry. 2014;13(1):12-20.

15. Kuek JHL, Raeburn T, Wand T. Asian perspectives on personal recovery in mental health: a scoping review. J Ment Health. 2020:1-17.

16. Tong A, Sainsbury P, Craig J. Consolidated criteria for reporting qualitative research (COREQ): a 32item checklist for interviews and focus groups. Int J Qual Health Care. 2007;19(6):349-57.

17. Flick U. Designing Qualitative Research. London: Sage Publications; 2007.

18. Kvale S. Doing Interviews. London: Sage Publications; 2007.

19. Barbour R. Doing Focus Groups. London: Sage Publications; 2007.

20. Gibbs GR. Analyzing Qualitative Data. London: Sage Publications; 2007.

21. Flick U. Managing Quality in Qualitative Research. London: Sage Publications; 2007.

22. Shanks V, Williams J, Leamy M, Bird VJ, Le Boutillier C, Slade M. Measures of personal recovery: a systematic review. Psychiatr Serv. 2013;64(10):974-80.

23. Liamputtong P. Research Methods in Health: Foundations for Evidence-Based Practice. Oxford: Oxford University Press; 2017.

24. Ajayi S, Billsborough J, Bowyer T, Brown P, Hicks A, Larsen J, et al. Getting back into the world: reflections on lived experiences of recovery. Rethink recovery series: volume 2,https://. Accessed 20 February 2021.

25. Lambert SD, Loiselle CG. Combining individual interviews and focus groups to enhance data richness. J Adv Nurs. 2008;62(2):228-37.

26. The Psychiatric Research Unit at the Mental Health Centre North Zealand. The WHO-5 website. About the WHO-5, https://www.psykiatri-regionh.dk/who-5/Pages/default.aspx. Accessed 20 February 2021.

27. Staehr Johansen K. The use of well-being measures in primary health care-the Dep-Care project; in World Health Organization, Regional Office for Europe: Well-Being Measures in Primary Health Care the DepCare Project. Geneva, World Health Organization,https://. Accessed 20 February 2021.

28. The Psychiatric Research Unit at the Mental Health Centre North Zealand. The WHO-5 website. WHO5 Questionnaires. WHO5 - Japanese, https://. Accessed 20 February 2021.

29. Awata S, Bech P, Yoshida S, Hirai M, Suzuki S, Yamashita M, et al. Reliability and validity of the Japanese version of the World Health Organization-Five Well-Being Index in the context of detecting 
depression in diabetic patients. Psychiatry Clin Neurosci. 2007;61(1):112-9.

30. Awata S, Bech P, Koizumi Y, Seki T, Kuriyama S, Hozawa A, et al. Validity and utility of the Japanese version of the WHO Five Well-Being Index in the context of detecting suicidal ideation in elderly community residents. Int Psychogeriatr. 2007;19(1):77-88.

31. Üstün TB, Kostanjsek N, Chatterji S, Rehm J, eds. Measuring Health and Disability: Manual for WHO Disability Assessment Schedule. WHODAS 2.0. Geneva: World Health Organization; 2010,https://apps.who.int/iris/handle/10665/43974. Accessed 20 February 2021.

32. World Health Organization, translated by M Tazaki, T Yamaguchi, Y Nakane. Measuring Health and

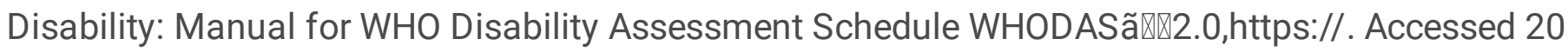
February 2021.

33. Gale NK, Heath G, Cameron E, Rashid S, Redwood S. Using the framework method for the analysis of qualitative data in multi-disciplinary health research. BMC Med Res Methodol. 2013;13:117.

34. Slade M, Rennick-Egglestone S, Blackie L, Llewellyn-Beardsley J, Franklin D, Hui A, et al. Posttraumatic growth in mental health recovery: qualitative study of narratives. BMJ Open. 2019;9(6):e029342.

35. Gelfand MJ, Raver JL, Nishii L, Leslie LM, Lun J, Lim BC, et al.ãudDifferences between tight and loose cultures: a 33-nation study. Science. 2011;332(6033):1100-4.

36. Mak WWS, Chan RCH, Yau SSW. Development and validation of Attitudes towards Recovery Questionnaire across Chinese people in recovery, their family carers, and service providers in Hong Kong. Psychiatry Res. 2018;267:48-55.

37. Markus HR, Kitayama S. Culture and the self: Implications for cognition, emotion, and motivation. Psychol Rev. 1991;98(2):224-53.

38. Uchida Y, Kitayama S, Mesquita B, Reyes JA, Morling B. Is perceived emotional support beneficial? Well-being and health in independent and interdependent cultures. Pers Soc Psychol Bull. 2008;34(6):741-54.

39. Ogihara Y, Uchida Y. Does individualism bring happiness? Negative effects of individualism on interpersonal relationships and happiness. Front Psychol. 2014;5:135.

40. Kasai K, Ando S, Kanehara A, Kumakura Y, Kondo S, Fukuda M, et al. Strengthening community mental health services in Japan. Lancet Psychiatry. 2017;4(4):268-70.

41. Bethell C, Jones J, Gombojav N, Linkenbach J, Sege R. Positive childhood experiences and adult mental and relational health in a statewide sample: associations across adverse childhood experiences levels. JAMA Pediatr. 2019;173(11):e193007.

42. Slade M, Oades L, Jarden A. Wellbeing, Recovery and Mental Health. Cambridge: Cambridge University Press; 2017.

43. Wong EC, Collins RL, Breslau J, Burnam MA, Cefalu MS, Roth E. Associations between provider communication and personal recovery outcomes. BMC Psychiatry. 2019;19(1):102. 
44. Repper J, Carter T. A review of the literature on peer support in mental health services. J Ment Health. 2011;20(4):392-411.

45. The best research is produced when researchers and communities work together. Nature. 2018;562(7725):7.

46. Sagiv L, Roccas S, Cieciuch J, Schwartz SH. Personal values in human life. Nat Hum Behav. 2017;1(9):630-9.

47. Kasai K, Fukuda M. Science of recovery in schizophrenia research: brain and psychological substrates of personalized value. NPJ Schizophr. 2017;3:14.

\section{Tables}

Due to technical limitations, tables are only available as a download in the Supplemental Files section.

\section{Supplementary Files}

This is a list of supplementary files associated with this preprint. Click to download.

- 210226RecoveryAdditionalfile1Kanehara.xIsx

- 210226RecoveryAdditionalfile2Kanehara.xlsx

- 210226RecoveryAdditionalfile3Kanehara.xlsx

- 210220RecoveryTable2aKanehara.xIsx

- 210220RecoveryTable2bKanehara.xIsx

- 210220RecoveryTable1Kanehara.xlsx 